\title{
Pattern Transfer of Laterally Aligned Lamellar Domains of Diblock Copolymers
}

\author{
Toru Yamaguchi and Hiroshi Yamaguchi \\ NTT Basic Research Laboratories, NTT Corporation \\ 3-1 Morinosato Wakamiya, Atsugi, Kanagawa 243-0198, Japan
}

Keywords: lithography, self-assembly, block copolymer, graphoepitaxy

\section{Introduction}

Block copolymer lithography (BCL) [1] has attracted considerable attention as a combined top-down/bottom-up approach to nanopatterning [2]. This method involves the use of microphaseseparated nanometer-sized domains as lithography templates [3-7]. The most important feature of this method is that the sizes and periods of the microphase-separated domains involved are determined solely by the chain length of the block copolymer. This feature makes BCL a promising candidate for high-resolution lithography at the 16-nm half-pitch node and beyond.

With regard to dot and hole patterns, many attempts have been made to apply these domains to the fabrication of quantum dots [8], patterned magnetic media $[9,10]$, silicon capacitors [11] FLASH memory [12], and so on. For the fabrication of nanodevices, dense line patterns are indispensable. Several research groups have successfully achieved dense line patterns by graphoepitaxy [13-16] or by using a chemically nanopatterned surface [17-19]. Independent of these works, we have already devised a novel method for the graphoepitaxy of the lamellar domains of symmetric diblock copolymers using resist patterns as alignment guides [20]. We have successfully demonstrated the lateral alignment of lamellar domains with a 16-nm half-pitch. For introducing block copolymer lithography into the semiconductor process, it is essential to confirm that these aligned domains can be transferred onto substrates.

In this study, we report the preliminary results of the pattern transfer of these laterally aligned lamellar domains with $16-\mathrm{nm}$ half-pitch by (a)

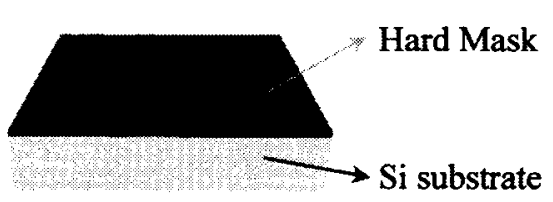

(b) PS domain PMMA domain

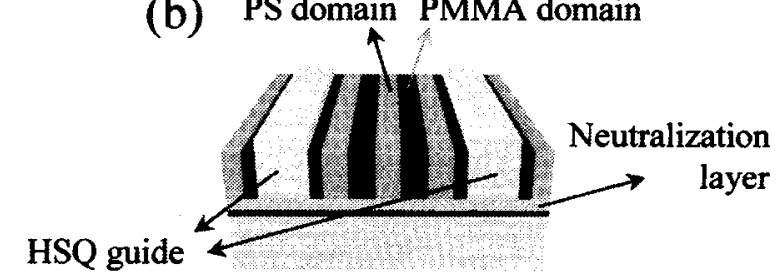

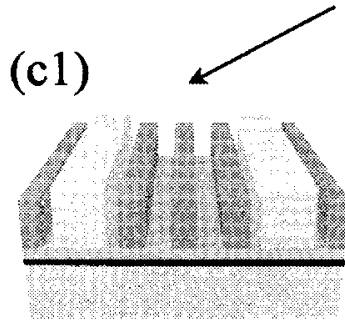

Process A
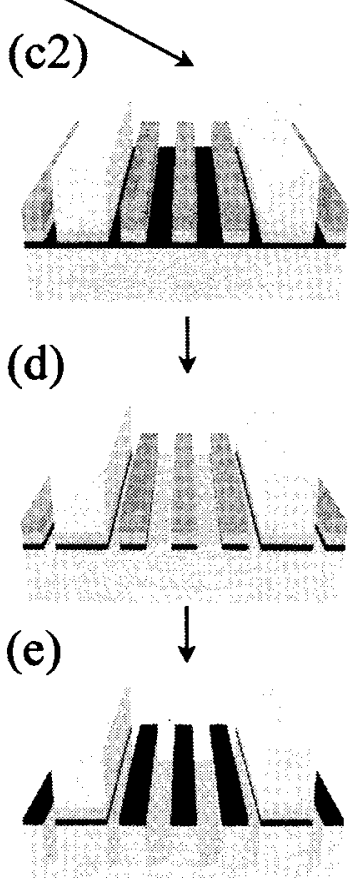

Process B
Fig.1 Schematic diagram of the fabrication process. 


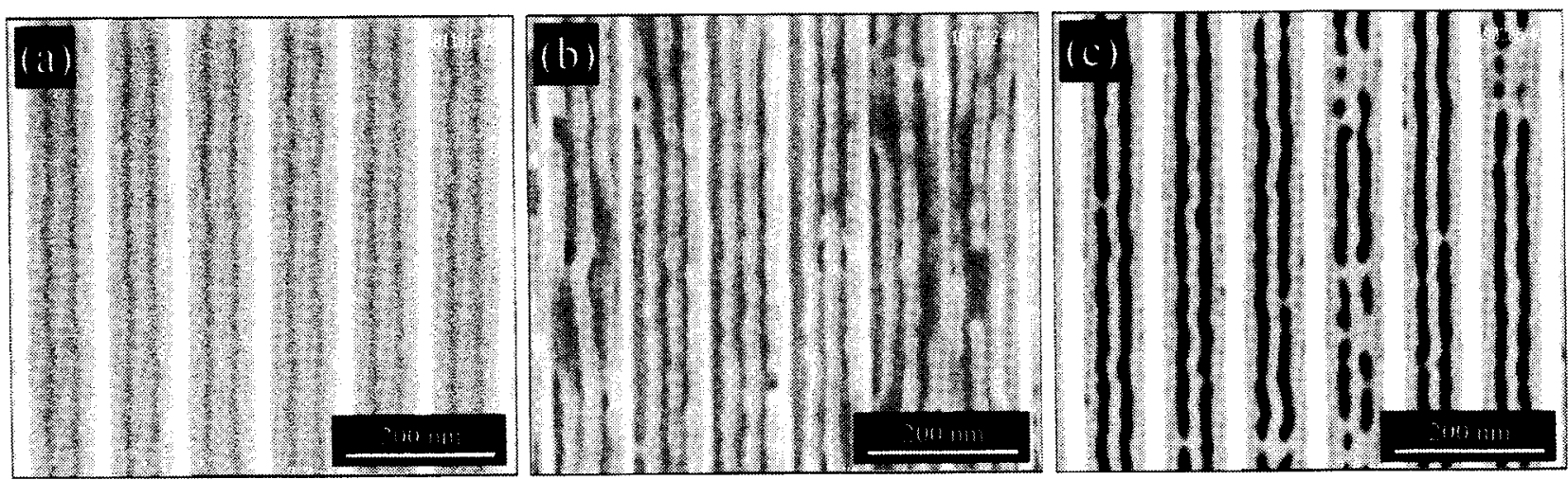

Fig. 2. Top-down SEM images of the microphase-separated domain morphology in $3 \mathrm{~L}_{0}$ confinement (a), the remaining PS patterns after the removal of the PMMA domains in Process A (b), and the etched patterns in Process $B$ (c). The bright white lines correspond to the HSQ guide patterns.

combining our alignment method and the hard-mask etching process.

\section{Fabrication Process}

Figure 1 shows a schematic diagram of the fabrication process employed in this approach. First, a 4-nm-thick silicon dioxide layer as a hard mask is formed on a Si substrate by exposing the substrate to oxygen plasma in a reactive ion-etching machine. Next, the lateral alignment of the lamellar domains of symmetric poly(styrene-b-methyl methacrylate) (lamellar period $\mathrm{L}_{0}=28 \mathrm{~nm}$ ) is achieved in confined spaces between hydrogen silsesquioxane (HSQ) resist patterns [21] on a neutral surface [22] by annealing the film in an oven at $195^{\circ} \mathrm{C}$ for $16 \mathrm{~h}$. The experimental details on the lateral alignment process have been described elsewhere [20].

In order to transfer the patterns from these laterally aligned domains onto a substrate, it is imperative that the PS domains are retained as an etching template by selectively removing the PMMA domains. There are two ways to remove the PMMA domains. One way is to expose the aligned domains to deep ultra violet (DUV) light and subsequently develop them with acetic acid (Process A). Another way is to expose them to oxygen plasma (Process B). The latter process utilizes the difference between the etching rates in the PS and PMMA domains. After the removal of the PMMA domains, the hard mask is etched with the remaining PS template using $\mathrm{CF}_{4}$-based gas chemistry. Finally, the Si substrate is etched with the hard-mask template using $\mathrm{Cl}_{2}$-based gas chemistry.

\section{Results and Discussion}

First, we describe the alignment of the lamellae between the straight guide patterns. Figure 2(a) shows a top-down SEM image of the microphase-separated domain morphology in $3 \mathrm{~L}_{0}$ confinement with a pitch of $110 \mathrm{~nm}$. It is clear that the two dark lines, which correspond to the PMMA domains, are distinctly separated from each other in all confinements. This indicates that lateral lamellae with a thickness of $3 \mathrm{~L}_{0}$ are perfectly aligned with the guide patterns in this confinement.

Next, we describe the removal of the PMMA domain using DUV exposure and the subsequent wet development process. Figure 2(b) shows a top-down SEM image of the aligned lamellar domains in $3 \mathrm{~L}_{0}$ confinement after the removal of the PMMA domains. It is clear that the remaining PS patterns collapse and remain stuck together. Although not shown here, all PS patterns diminish in some places. There should be at least two reasons for this pattern deformation. One is the pattern collapse induced by the surface tension of water during the drying step. The other is the poor adhesion of the PS patterns to the bottom neutralization layer. The neutralization layer functions as a cross-linked positive-tone resist. Cross-linked polymers are fragmented by DUV exposure and then adhesion becomes weak. As a result, some PS patterns lift off during the development step. From the viewpoint of pattern transfer, therefore, the wet process is not adequate for the removal of the PMMA domains.

Next, we describe pattern transfer by using the dry development process. Figure 2(c) shows a top-down SEM image of the etched patterns in $3 \mathrm{~L}_{0}$ confinement. It is clear that two the PMMA domains observed in the surface morphology [Fig. 2(a)] are successfully transferred for all confinements. However, many bridges are observed 
in the etched patterns. The reason why these bridges are formed is presently unclear and requires further investigation.

\section{Conclusion}

We investigated the pattern transferability of laterally aligned lamellar domains in confined spaces between resist patterns as alignment guides. The results confirmed that all dry etching processes are strongly recommended for pattern transfer from aligned lamellar domains. We provided a preliminary demonstration of the fabrication of 16-nm half-pitch dense lines by pattern transfer from aligned lateral lamellar domains. We believe that our approach can be applied to the fabrication of dense lines at around the 16-nm technology node.

\section{Acknowledgements}

We would like to thank Junzo Hayashi for the sample preparation, Kazuhito Inokuma and Dr. Kenji Yamazaki for EB exposure. This work was partly supported by JSPS KAKENHI (16206003).

\section{References}

1. M. Park, C. Harrison, P. M. Chaikin, R. A. Register, and D. H. Adamson, Science 276, 1401 (1997).

2. R. A. Segalman, H. Yokoyama, and E. J. Kramer, Adv. Mater. 13, 1152 (2001).

3. C. Harrison, M. Park, P. M. Chaikin, R. A. Register, D. H. Adamson, J. Vac. Sci. Technol. B16 544 (1998).

4. T. Thurn-Albrecht, R. Steiner, J. DeRouchey, C. M. Stafford, E. Huang, M. Bal, M. Tuominen, C. J. Hawker, and T. P. Russell, Adv. Mater. 12, 787 (2000).

5. H.-C. Kim, X. Jia, C. M. Stafford, D. H. Kim, T. J. McCarthy, M. Tuominen, C. J. Hawker, and T. P. Russell, Adv. Mater. 13, 795 (2001).

6. J. Y. Cheng, C. A. Ross, V. Z.-H. Chan, E. L. Thomas, R. G. H. Lammertink, and G. J. Vansco, Adv. Mater. 13, 1174 (2001).
7. K. W. Guarini, C. T. Black, K. R. Milkove, and R. L. Sandstorm, J. Vac. Sci. Technol. B19, 2784 (2001).

8. R. R. Li, P. D. Dapkus, M. E. Thompson, W. G. Jeong, C. Harrison, P. M. Chaikin, R. A. Register, D. H. Adamson, Appl. Phys. Lett. 76, 1689 (2000).

9. K. Asakawa, T. Hiraoka, H. Hieda, M. Sakurai, Y. Kamata, and K. Naito, J. Photopolym. Sci. Technol. 15, 465 (2002).

10. J. Y. Cheng, C. A. Ross, E. L. Thomas, H. I. Smith, and G. J. Vansco, Appl. Phys. Lett. 81, 3657 (2002).

11. C. T. Black, K. W. Guarini, K. R. Milkove, S. M. Baker, T. P. Russell, and M. T. Tuominen, Appl. Phys. Lett. 79, 409 (2001).

12. K. W. Guarini, C. T. Black, Y. Zhang, I. V. Babich, E. M. Sikorski, and L. M. Gignac, International Electron Devices Meetings, Technical Digest 2003, 541.

13. D. Sundrani, S. B. Darling, and S. J. Sibener, Nano Lett. 4, 273 (2004).

14. H. -W. Li and W. T. S. Huck, Nano Lett., 4, 1633 (2004).

15. C. T. Black, and O. Bezencenet, IEEE Trans. Nanotechnol. 3, 412 (2004).

16. S-M. park, M. P. Stoykovich, R. Ruiz, Y. Zhang, C. T. Black, and P. F. Nealey, Adv. Mater. 19, 607 (2007).

17. S. O. Kim, H. H. Solak, M. P. Stoykovich, N. J. Ferrier, J. J. de Pablo, and P. F. Nealey, Nature 424 (2003) 411.

18. M. P. Stoykovich, M. Muller, S. O. Kim, H. H. Solak, E. W. Edwards, J. J. de Pablo, and P. F. Nealey, Science 308 (2005) 1442.

19. R. Ruiz, R. L. Sandstrom, and C. T. Black, $A d v$. Mater. 19, 587 (2007).

20. T. Yamaguchi, and H. Yamaguchi, $J$. Photopolym. Sci. Technol. 19, 385 (2006).

21. H. Namatsu, Y. Takahashi, K. Yamazaki, T. Yamaguchi, M. Nagase, and K. Kurihara., J. Vac. Sci. Technol., B16 (1998) 69.

22. P. Mansky, Y. Liu, E. Huang, T. P. Russel, and C. Hawker, Science 275 (1997) 1458. 